\title{
Vertex renormalization and hard scattering symmetry breaking corrections to $B$ to axial vector meson form factors at large recoil
}

\author{
Arslan Sikandar@, ${ }^{1}$ M. Jamil Aslam, ${ }^{1,2}$ Ishtiaq Ahmed, ${ }^{3}$ and Saba Shafaq ${ }^{4}$ \\ ${ }^{1}$ Physics Department, Quaid-i-Azam University, Islamabad 45320, Pakistan \\ ${ }^{2}$ Physics Department, University of Alberta, Edmonton T6G2E1, Alberta, Canada* \\ ${ }^{3}$ National Centre for Physics, Quaid-i-Azam University Campus, Islamabad 45320, Pakistan \\ ${ }^{4}$ Department of Physics, International Islamic University (IIU), Islamabad 44000, Pakistan
}

(Received 16 May 2019; published 20 September 2019)

\begin{abstract}
The symmetries arise due to heavy quarks, and the large energy limit helps us to reduce the number of independent form factors in the heavy-to-light $B$-meson decays. It is expected that these symmetry relations are not exact and that they are broken by the perturbative effects, namely, the vertex corrections and the hard-spectator scatterings. The former are included in the form factors via vertex renormalization, whereas the latter are calculated through light-cone distribution amplitudes. We first calculate these symmetry breaking corrections to the form factors involved in semileptonic $B$-meson to an axial-vector $\left(K_{1}\right)$-meson decay. Later, by using these form factors we see their effect on the physical observables such as the zero position of the forward-backward $\left(\mathcal{A}_{F B}\right)$ asymmetry and the longitudinal lepton polarization $\left(P_{L}\right)$ asymmetry in $B \rightarrow K_{1}(1270) \mu^{+} \mu^{-}$decay. We find that as a result of these corrections to the form factors, the zero position of the forward-backward asymmetry is shifted by $10 \%$ from its SM value, while the effects on $P_{L}$ are rather insignificant.
\end{abstract}

DOI: $10.1103 /$ PhysRevD.100.056013

\section{INTRODUCTION}

Form factors for a $B$ meson decaying to a light meson $f$, where $f$ can be a pseudoscalar $(P)$, a vector $(V)$, an axialvector $(A)$, or a tensor $(T)$ meson, arise due to the matrix element of local flavor-changing currents (FCC) $\bar{q} \Gamma q$, with $\Gamma$ representing some spin structure. The hadronic form factors play a crucial role in the accurate predictions of some physical observables (e.g., branching ratios, angular coefficients, etc.,) in different semileptonic $B$-meson decays. At present, several measurements of $B$ decays involving both flavor-changing neutral currents (FCNC) $b \rightarrow(d, s) \ell^{+} \ell^{-}$and flavor-changing charged currents $\left(b \rightarrow c \ell \nu_{\ell}\right)$ have shown the possible hints of physics beyond the standard model (SM) (see, e.g., [1,2] and references therein). Despite the accurately known form factors for $B \rightarrow K^{*}$ and $B \rightarrow D^{*}$, the efforts to make them more precise are still the focus of ongoing theoretical and phenomenological studies [3].

In heavy-to-light decays, the form factors are mainly dominated by the quantum chromodynamics (QCD)

\footnotetext{
${ }^{*}$ Visiting professor.
}

Published by the American Physical Society under the terms of the Creative Commons Attribution 4.0 International license. Further distribution of this work must maintain attribution to the author(s) and the published article's title, journal citation, and DOI. Funded by SCOAP. interactions at small momentum transfer, and hence they cannot be computed in perturbation theory. Effective theories embedding certain symmetries are used to reduce the number of independent form factors. In this context, one of the symmetries in the decays of mesons containing heavy quarks is known as heavy-quark symmetry (HQS), which is based on an expansion in inverse powers of the heavy-quark mass. By invoking this symmetry expansion, one can get certain symmetry relations [4-6] which may not be evident in the full QCD. These relations are used to relate the matrix elements corresponding to different currents and hence reduce the number of independent form factors [7-9]. For instance, in $B \rightarrow D^{*}$ the seven independent form factors can be reduced to a single Isgur Wise function [4].

In the case of semileptonic decays $B \rightarrow\left(\pi, \rho, K^{*}\right) \ell^{+} \ell^{-}$, when the energy $(E)$ of the final state meson is large, one can make the expansion in the powers of $1 / E$, and the resulting theory is known as the large-energy effective theory (LEET) [7]. Using the HQS for the initial-state $B$ meson and LEET for the final-state light meson, one can factorize the form factors in soft and hard parts. The soft part of the form factors accounts for the soft gluon interaction with the spectator, while the hardspectator interactions are carried out by gluons having a momentum of the order of $m_{B} \Lambda_{\mathrm{QCD}}$, where $m_{B}$ is the mass of the initial-state $B$ meson. These contributions are not independent of each other, and in the case of 
$B \rightarrow \rho\left(K^{*}\right) \ell^{+} \ell^{-}$decays, it is shown that in the LEET the seven form factors reduce to two in the large recoil limit [10]. However, these symmetry relations are not exact, and they are broken by the radiative corrections. These symmetry breaking corrections at first order in the strong coupling constant $\alpha_{s}$ were computed by Beneke and Feldmann [10], along with their implications to the forward-backward asymmetry in $B \rightarrow \rho \ell^{+} \ell^{-}$decays. Later, these radiative corrections for $B \rightarrow K^{*} l^{+} l^{-}$were calculated in Ref. [11].

A close kin of the FCNC mediated $B \rightarrow K^{*} \ell^{+} \ell^{-}$decay is $B \rightarrow K_{1}(1270,1400) \ell^{+} \ell^{-}$, where $K_{1}(1270,1400)$ are the axial-vector mesons. These axial-vector states are a mixture of the members of two axial-vector $S U(3)$ octet ${ }^{3} P_{1}$ and ${ }^{1} P_{1}$ states, $K_{1 A}$ and $K_{1 B}$, respectively. The physical states $K_{1}(1270)$ and $K_{1}(1400)$ are related to flavor states $K_{1 A}$ and $K_{1 B}$ as

$$
\left(\begin{array}{c}
\left|K_{1}(1270)\right\rangle \\
\left|K_{1}(1400)\right\rangle
\end{array}\right)=\left(\begin{array}{cc}
\sin \theta_{K_{1}} & \cos \theta_{K_{1}} \\
\cos \theta_{K_{1}} & -\sin \theta_{K_{1}}
\end{array}\right)\left(\begin{array}{l}
\left|K_{1, A}\right\rangle \\
\left|K_{1, B}\right\rangle
\end{array}\right),
$$

where $\theta_{K_{1}}$ is the mixing angle, and its value, estimated from the radiative $B \rightarrow K_{1}(1270) \gamma$ and $\tau \rightarrow K_{1}(1270) \nu_{\tau}$ decays, is $-(34 \pm 13)^{o}$ [12]. It is worth emphasizing at this point that the above unitary matrix is also used to relate all the parameters of the $K_{1 A, B}$ and physical $K_{1}(1270,1400)$ states.

Now, being mediated through the quark level transition, $b \rightarrow s \ell^{+} \ell^{-}$, the effective Hamiltonian remains the same in $B \rightarrow K^{*} \ell^{+} \ell^{-}$and $B \rightarrow K_{1}(1270,1400) \ell^{+} \ell^{-}$ decays. Hence, the constraints on the Wilson coefficients and other parameters obtained by analyzing different new physics (NP) scenarios in $B \rightarrow K^{*} \ell^{+} \ell^{-}$can also be used to find the complementary information from $B \rightarrow$ $K_{1}(1270,1400) \ell^{+} \ell^{-}$decays. Due to this fact, a thorough analysis of this decay has been done in different NP scenarios (see, e.g., [13-19] and references therein). Despite rigorous NP studies in $B \rightarrow K_{1}(1270,1400) \ell^{+} \ell^{-}$ decays, the contributions arising from the symmetry breaking corrections to the form factors are still missing in the literature, and the main motivation of the present study is to fill this gap. In order to achieve this goal, we follow a factorization scheme developed in [10] that factorizes the soft and hard contributions of the form factors in the framework of the LEET. The corrections to the soft part are manifested in the Wilson coefficients at an order $\alpha_{s}$ by matching the LEET calculation with the full one-loop QCD calculation, while for the hardspectator part, the nonperturbative method is required, for which we use light-cone distribution amplitudes (LCDA). These hard-spectator corrections actually break the symmetry relations. At large recoil, a significant amount of energy is taken by the final-state leading light quark, for which an expansion over energy is a viable approach. For a more probable final meson state, in which both the leading light and spectator quarks share similar momenta, hard gluon interactions become more and more important. The calculation of hardspectator corrections along with the vertex renormalization in $B \rightarrow K_{1}(1270)$ is the main objective of this study. After quantifying these corrections, their impact on the physical observables that are known to have less dependence on the form factors, namely, the zero position of the forward-backward asymmetry and the longitudinal lepton polarization asymmetry, are studied for $B \rightarrow K_{1}(1270) \mu^{+} \mu^{-}$. The case where the final-state meson is $K_{1}(1400)$ is rather trivial.

The paper is organized as follows: In Sec. II, after a brief introduction of the LEET, the Lagrangian will be given by keeping the final meson mass terms that respect HQS. The seven form factors for $B \rightarrow K_{1}$, where from here on $K_{1}$ refers to $K_{1}(1270)$, transitions are written in terms of the two soft form factors $\xi_{K_{1}}^{\perp, \|}\left(E_{F}\right)$ using LEET symmetries. In Sec. III, we describe the factorization scheme used to calculate the symmetry breaking corrections to the form factors. The vertex renormalization is carried out along with the hard-spectator interactions to calculate the symmetry breaking corrections at an order $\alpha_{s}$. The major uncertainties in the calculation of the form factors lie in hard-spectator corrections, especially in the $B$-meson distribution amplitudes. This is discussed in Sec. IVA. Using light-cone sum rules (LCSR) form factors [20,21], both without corrections and by incorporating the radiative corrections, are discussed in the same section. Later, their impact on the forwardbackward and the longitudinal lepton polarization asymmetries is studied in Sec. IV B. We conclude in Sec. V. The study presented here is supplemented with three appendixes: Appendix A summarizes the $B$ - and $K_{1^{-}}$ meson distribution amplitudes, and Appendix B presents the expressions of different helicity amplitudes for the decay under consideration. Finally, Appendix $\mathrm{C}$ gives the detailed calculation of the hard-spectator correction to one of the form factors $V_{2}\left(q^{2}\right)$.

\section{FORM FACTORS AT LARGE RECOIL}

The matrix elements for the decay of the $B$ meson to an axial-vector meson $\left(K_{1}\right)$ can be written as 


$$
\begin{aligned}
\left\langle K_{1}\left(p^{\prime}, \varepsilon^{*}\right)\left|\bar{q} \gamma^{\mu} b\right| \bar{B}(p)\right\rangle= & 2 m_{K_{1}} V_{0}\left(q^{2}\right) \frac{\varepsilon^{*} \cdot q}{q^{2}} q^{\mu}+\left(m_{B}+m_{K_{1}}\right) V_{1}\left(q^{2}\right)\left\{\varepsilon^{* \mu}-\frac{\varepsilon^{*} \cdot q}{q^{2}} q^{\mu}\right\} \\
& -V_{2}\left(q^{2}\right) \frac{\varepsilon^{*} \cdot q}{m_{B}+m_{K_{1}}}\left\{\left(p+p^{\prime}\right)^{\mu}-\frac{m_{B}^{2}-m_{K_{1}}^{2}}{q^{2}} q^{\mu}\right\} \\
\left\langle K_{1}\left(p^{\prime}, \varepsilon^{*}\right)\left|\bar{q} \gamma^{\mu} \gamma_{5} b\right| \bar{B}(p)\right\rangle= & \frac{2 i A\left(q^{2}\right)}{m_{B}+m_{K_{1}}} \epsilon^{\mu \nu \rho \sigma} \varepsilon_{\nu}^{*} p_{\rho}^{\prime} p_{\sigma} \\
\left\langle K_{1}\left(p^{\prime}, \varepsilon^{*}\right)\left|\bar{q} \sigma^{\mu \nu} q_{\nu} \gamma_{5} b\right| \bar{B}(p)\right\rangle= & -2 T_{1}\left(q^{2}\right) \epsilon^{\mu \nu \rho \sigma} \varepsilon_{\nu}^{*} p_{\rho}^{\prime} p_{\sigma} \\
\left\langle K_{1}\left(p^{\prime}, \varepsilon^{*}\right)\left|\bar{q} \sigma^{\mu \nu} q_{\nu} b\right| \bar{B}(p)\right\rangle= & -i T_{2}\left(q^{2}\right)\left\{\left(m_{B}^{2}-m_{K_{1}}^{2}\right) \varepsilon^{* \mu}-\left(\varepsilon^{*} \cdot q\right)\left(p+p^{\prime}\right)^{\mu}\right\} \\
& -i T_{3}\left(q^{2}\right)\left(\varepsilon^{*} \cdot q\right)\left\{q^{\mu}-\frac{q^{2}}{m_{B}^{2}-m_{K_{1}}^{2}}\left(p+p^{\prime}\right)^{\mu}\right\}
\end{aligned}
$$

where $p^{\mu}\left(p^{\prime \mu}\right)$ and $m_{B}\left(m_{K_{1}}\right)$ are the momentum and mass of the $B\left(K_{1}\right)$ meson, respectively, and $\varepsilon^{* \mu}$ is the polarization vector of the $K_{1}$ meson. The interaction of heavy quarks with soft gluons results in heavy-quark effective theory (HQET) [6]. Using $q^{2}=\left(p-p^{\prime}\right)^{2}$, the energy of the final-state $K_{1}$ meson is $E_{F}=\left(m_{B}^{2}+m_{K_{1}}^{2}-q^{2}\right) / 2 m_{B}$. In the large recoil region, the momentum transfer squared, i.e., $q^{2}$, is small, and since $m_{K_{1}}^{2} \ll m_{B}^{2}$ and $q^{2} \ll m_{B}^{2}$, the energy of the $K_{1}$ meson is of order $E_{F} \sim m_{B} / 2$. Therefore, $E_{F}$ is a good expansion parameter, and for the $K_{1}$ meson whose mass is larger than $1 \mathrm{GeV}$, retaining terms of the order of $m_{K_{1}}^{2} / m_{B}^{2}$ would be interesting. However, it is safe to neglect the terms of the order of $\Lambda_{\mathrm{QCD}} / E_{F}$.

The four-momentum of the $B$ meson in terms of the velocity $v$ of the heavy quark can be expressed as $p^{\mu}=m_{B} v^{\mu}$. Being a bound state of a heavy and a light quark, the heavy quark has momentum

$$
p_{Q}^{\mu}=m_{Q} v^{\mu}+k^{\mu},
$$

where $k$ is the residual momentum and $|k| \sim \Lambda_{\mathrm{QCD}} \ll m_{b}$. In the $B$-meson rest frame, the components of the fourvelocity are $v=(1,0,0,0)$. In order to work with lightcone variables, let us introduce two lightlike vectors $n_{\mu}$ and $\eta_{\mu}$ satisfying $n^{2}=\eta^{2}=0$. Choosing $n=(1,0,0,1)$ and $\eta=(1,0,0,-1)$, it can be verified that $\eta_{\mu}=2 v_{\mu}-n_{\mu}$ while $n \cdot v=1$ and $n \cdot \eta=2$.

The momentum of the $K_{1}$ meson in terms of $n_{\mu}$ and $\eta_{\mu}$ can be expressed as [22]

$$
p_{F}=E n+\frac{m_{K_{1}}^{2}}{4 E} \eta
$$

with $E$ being the off-shell energy. The on-shell energy and three-momentum of the final-state $K_{1}$ meson are

$$
E_{F}=E\left(1+\frac{m_{K_{1}}^{2}}{4 E^{2}}\right), \quad \Delta \equiv \sqrt{E_{F}^{2}-m_{K_{1}}^{2}}=E\left(1-\frac{m_{K_{1}}^{2}}{4 E^{2}}\right),
$$

and the momentum of the leading light quark in the final state is given as

$$
p_{q}=E n+\frac{m_{K_{1}}^{2}}{4 E} \eta+k^{\prime}=\Delta n+\frac{m_{K_{1}}^{2}}{4 E} v+k^{\prime},
$$

where again the residual momentum is of the order of $\left|k^{\prime}\right| \sim \Lambda_{\mathrm{QCD}} \ll E$. The effective Lagrangian can be derived from the four-component QCD quark fields $q(x)$. The twocomponent light-quark fields are given via projection operators $\left(P_{ \pm}\right)$as

$$
q_{ \pm}(x)=\exp \left(i \Delta \cdot x+\frac{m_{q}^{2}}{2 E} v \cdot x\right) P_{ \pm} q(x),
$$

with

$$
\begin{array}{ll}
P_{+}=\frac{\not h \phi}{2}, & P_{+}^{2}=P_{+}, \\
P_{-}=\frac{\not h h}{2}, & P_{-}^{2}=P_{-} .
\end{array}
$$

The effective Lagrangian up to order $\Lambda_{\mathrm{QCD}} / E$ while retaining terms of order $m_{K_{1}}^{2} / E$ can be expressed by

$$
\mathcal{L}=\bar{q}(x) \not\left(\text { in } \cdot D+\frac{m_{K_{1}}^{2}}{2 E}\right) q(x),
$$

where $D^{\mu}=\partial^{\mu}-i g_{s} A^{\mu}$ is the covariant derivative. The second term in the Lagrangian gives the contribution due to the final-state meson mass, and it does not break the symmetry of the leading-order Lagrangian due to similar Dirac structure. In the case of the vector-meson $\left(\rho, K^{*}\right)$, whose mass is less than $1 \mathrm{GeV}$, the terms of the order $\frac{m_{K^{*}}^{2}}{2 E}$ are ignored in $B \rightarrow\left(\rho, K^{*}\right) \ell^{+} \ell^{-}$calculations $[10,11]$. 
In our calculation of form factors of a $B$ meson decaying to a $K_{1}$ meson, it is instructive to define the sum and difference of momenta,

$$
\begin{array}{r}
\left(p+p^{\prime}\right)^{\mu}=m_{B}\left(1+\frac{m_{K_{1}}^{2}}{2 m_{B} E}\right) v^{\mu}+\Delta n^{\mu}, \\
q \equiv\left(p-p^{\prime}\right)^{\mu}=m_{B}\left(1-\frac{m_{K_{1}}^{2}}{2 m_{B} E}\right) v^{\mu}-\Delta n^{\mu} .
\end{array}
$$

The $K_{1}$ meson with polarization vector $\varepsilon^{* \mu}$ satisfies the transverse relation, i.e., $\varepsilon^{*} \cdot p^{\prime}=0$. From Eq. (10), contracting with $\varepsilon^{* \mu}$ and making use of the transverse relation, we have a useful identity,

$$
\varepsilon^{*} \cdot n=-\frac{m_{K_{1}}^{2}}{2 E \Delta}\left(\varepsilon^{*} \cdot v\right) .
$$

Using the technique from HQET, the soft form factors relation can be found as [6]

$$
\left\langle K_{1}\left(p^{\prime}, \varepsilon^{*}\right)|\bar{q} \Gamma b| B(p)\right\rangle=\operatorname{Tr}\left[A\left(E_{F}\right) \bar{M}_{K_{1}} \Gamma M_{B}\right],
$$

where the projector for the $K_{1}$ meson is defined as $\bar{M}_{K_{1}}=$ $-\varepsilon^{*} \gamma_{5} \frac{1 \|}{2}$ and for the $B$ meson, it is $M_{B}=-\frac{1+\gamma}{2} \gamma_{5}$. The function $A\left(E_{F}\right)$ contains the long-distance dynamics that is independent of any Dirac structure $\Gamma$, and it can be written as

$$
A\left(E_{F}\right)=E_{F} \not h\left\{\xi_{K_{1}}^{\perp}\left(E_{F}\right)-\frac{\not}{2} \xi_{K_{1}}^{\|}\left(E_{F}\right)\right\},
$$

where $\xi_{K_{1}}^{\perp}\left(E_{F}\right)$ and $\xi_{K_{1}}^{\|}\left(E_{F}\right)$ denote the contribution to form factors from transversely and longitudinally polarized $K_{1}$ mesons, respectively. Substituting $M_{K_{1}}, M_{B}$ along with the function $A\left(E_{F}\right)$ in Eq. (12) and considering the possible Dirac structures $\Gamma=\left\{\gamma^{\mu}, \gamma^{\mu} \gamma_{5}, \sigma^{\mu \nu} q_{\nu}, \sigma^{\mu \nu} q_{\nu} \gamma_{5}\right\}$, the trace calculation gives

$$
\begin{aligned}
\left\langle K_{1}\left(p^{\prime}, \varepsilon^{*}\right)\left|\bar{q} \gamma^{\mu} b\right| B(p)\right\rangle= & 2 E_{F} \xi_{K_{1}}^{\perp}\left(E_{F}\right)\left[\varepsilon^{* \mu}-\left(\varepsilon^{*} \cdot v\right)\left(\frac{E_{F}}{\Delta} n^{\mu}-\frac{m_{K_{1}}^{2}}{2 E \Delta} v^{\mu}\right)\right] \\
& +2 E_{F}\left(1+\frac{m_{K_{1}}^{2}}{4 E \Delta}\right) \xi_{K_{1}}^{\|}\left(E_{F}\right)\left(\varepsilon^{*} \cdot v\right) n^{\mu}, \\
\left\langle K_{1}\left(p^{\prime}, \varepsilon^{*}\right)\left|\bar{q} \gamma^{\mu} \gamma_{5} b\right| B(p)\right\rangle= & 2 i E_{F} \xi_{K_{1}}^{\perp}\left(E_{F}\right) \varepsilon^{\mu \nu \rho \sigma} \varepsilon_{\nu}^{*} n_{\rho} v_{\sigma}, \\
\left\langle K_{1}\left(p^{\prime}, \varepsilon^{*}\right)\left|\bar{q} \sigma^{\mu \nu} \gamma_{5} q_{\nu} b\right| B(p)\right\rangle= & 2 E_{F} m_{B} \xi_{K_{1}}^{\perp}\left(1-\frac{m_{K_{1}}^{2}}{2 E m_{B}}\right) \varepsilon^{\mu \nu \rho \sigma} \varepsilon_{\nu}^{*} n_{\rho} v_{\sigma}, \\
\left\langle K_{1}\left(p^{\prime}, \varepsilon^{*}\right)\left|\bar{q} \sigma^{\mu \nu} q_{\nu} b\right| B(p)\right\rangle= & 2 i E_{F}\left[m_{B} \xi_{K_{1}}^{\perp}\left(E_{F}\right)\left(1-\frac{m_{K_{1}}^{2}}{2 E m_{B}}\right)\left\{\varepsilon^{* \mu}-\varepsilon^{*} \cdot v\left(\frac{E_{F}}{\Delta} n^{\mu}-\frac{m_{K_{1}}^{2}}{2 E \Delta} v^{\mu}\right)\right\}\right. \\
& \left.+\frac{E}{\Delta} \xi_{K_{1}}^{\|}\left(E_{F}\right)\left(\varepsilon^{*} \cdot v\right)\left\{\left(m_{B}-E_{F}\right) n^{\mu}-m_{B}\left(1-\frac{m_{K_{1}}^{2}}{2 E m_{B}}\right) v^{\mu}\right\}\right] .
\end{aligned}
$$

It is important to emphasis that despite the fact that both $B \rightarrow K^{*} \ell^{+} \ell^{-}$and $B \rightarrow K_{1} \ell^{+} \ell^{-}$decays are mediated by the quark level transition $b \rightarrow s \ell^{+} \ell^{-}$, there are some differences. The first and the obvious difference is that $K_{1}$ is an axial-vector meson; due to this fact, the matrix elements corresponding to vector and axial-vector currents in the $K^{*}$ case are interchanged here, and this can be seen in Eq. (2). The second common difference between $K^{*}$ and $K_{1}(1270,1400)$ is that the latter states are a mixture of flavor eigenstates $K_{1 A, 1 B}$, and hence the corresponding form factors and other quantities will also mix, which is not the case for the $K^{*}$ meson. The last and the most particular one is that contrary to the $K^{*}$ meson, the mass of the $K_{1}$ meson is above $1 \mathrm{GeV}$, and hence, without ignoring its mass, such symmetry relations for form factors were calculated in [22]. Therefore, we have also kept the $K_{1}$ meson mass terms in calculating vertex and hard-spectator corrections. By ignoring the final-state meson mass in these correction terms and also interchanging the role of vector and axial-vector currents, one can see that the corresponding relations for the $B \rightarrow K^{*} \ell^{+} \ell^{-}$can be reproduced.

Now comparing Eq. (2) with Eq. (14) to represent all seven form factors in terms of the two soft form factors $\xi_{K_{1}}^{\perp, \|}\left(E_{F}\right)$, one gets

$$
\begin{gathered}
V_{0}\left(q^{2}\right)=\frac{E_{F}}{m_{K_{1}}} \xi_{K_{1}}^{\|}\left(E_{F}\right), \\
V_{1}\left(q^{2}\right)=\frac{2 E_{F}}{m_{B}+m_{K_{1}}} \xi_{K_{1}}^{\perp}\left(E_{F}\right), \\
V_{2}\left(q^{2}\right)=\left(1+\frac{m_{K_{1}}}{m_{B}}\right)\left(1+\frac{2 m_{K_{1}}^{2}}{m_{B}^{2}}\right)\left[\xi_{K_{1}}^{\perp}\left(E_{F}\right)-\xi_{K_{1}}^{\|}\left(E_{F}\right)\right],
\end{gathered}
$$




$$
\begin{gathered}
A\left(q^{2}\right)=\left(1+\frac{m_{K_{1}}}{m_{B}}\right) \frac{E_{F}}{\Delta} \xi_{K_{1}}^{\perp}\left(E_{F}\right) \\
T_{1}\left(q^{2}\right)=\left(1-\frac{m_{K_{1}}^{2}}{m_{B}^{2}}\right) \frac{E_{F}}{\Delta} \xi_{K_{1}}^{\perp}\left(E_{F}\right) \\
T_{2}\left(q^{2}\right)=\frac{2 E_{F}}{m_{B}} \xi_{K_{1}}^{\perp}\left(E_{F}\right) \\
T_{3}\left(q^{2}\right)=\left(1+\frac{5 m_{K_{1}}^{2}}{m_{B}^{2}}\right) \xi_{K_{1}}^{\perp}\left(E_{F}\right)-\left(1+\frac{2 m_{K_{1}}^{2}}{m_{B}^{2}}\right) \xi_{K_{1}}^{\|}\left(E_{F}\right)
\end{gathered}
$$

Recall that the energy $E_{F}$ is a function of $q^{2}$ and for the radiative decays $q^{2}=0$; therefore, we get the trivial expressions for the form factors. The form factor $V_{0}\left(q^{2}\right)$ only depends upon $\xi_{K_{1}}^{\|}\left(E_{F}\right)$, and it will be used later as a renormalization convention along with $A\left(q^{2}\right)$ for the perpendicular-polarization form factor $\xi_{K_{1}}^{\perp}\left(E_{F}\right)$.

\section{SYMMETRY BREAKING CORRECTIONS}

The symmetries that arise in the HQET help us to relate the form factors and hence reduce the number of independent form factors, e.g., from seven to two in the decay under consideration. However, the heavy-quark-largeenergy symmetries are broken by the radiative corrections. These corrections arise from a vertex diagram as shown in Fig. 1 or from the hard-spectator scattering diagrams shown in Fig. 2. For HQS at large recoil, we worked out the relations of the soft form factors in Sec. II. The vertex contributions arise at the order of $1 / m_{B}$ and $\alpha_{s}$. The contributions arising from hard-spectator interactions are suppressed by an order of $\alpha_{s}$. However, in the case of heavy-to-light transitions, these corrections are still important. This is due to the fact that we desire a probable

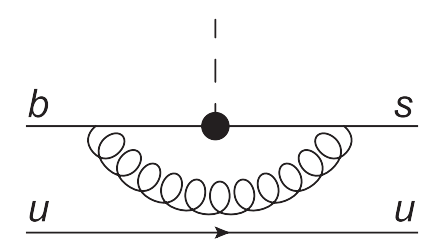

FIG. 1. Vertex correction in $B \rightarrow K_{1}$ decays.

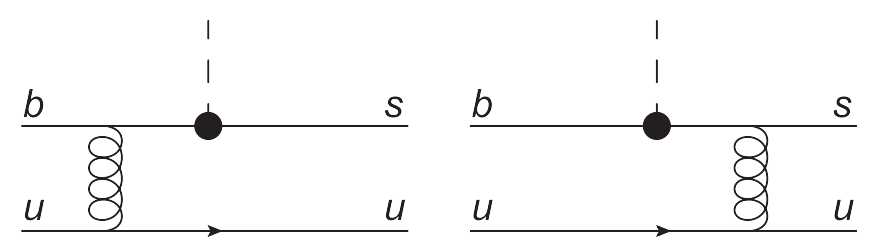

FIG. 2. Hard spectator corrections to the form factors in $B \rightarrow K_{1}$ decays. configuration in which the momentum of the spectator and leading quark scale in a similar fashion. This requires a well-thought-out factorization scheme, and to serve this purpose, there have been a few factorization schemes developed in the last 10-15 years for these heavy-to-light transitions (e.g., [10,23]). Beneke and Feldmann [10] have developed a factorization scheme in terms of soft and hard contributions to the form factors in the framework of LEET, and we adopt it in our present work. We see that the vertex corrections do not respect the symmetry relations, and to take them into account, these are calculated at an extra order of $\alpha_{s}$ in an effective theory and then matched with full theory. The difference in matching the two will give us the required contributions. There is one subtlety: LEET is not infrared safe because it does not take care of the collinear gluons and hence cannot correctly reproduce infrared divergences. However, this will be studied in a future work [24]. But in the context of this work, such collinear gluons do not break symmetry relations among the soft form factors [25]. Similarly, for the hard-spectator scattering, it is seen that end-point divergences respect the HQS and are also accounted for in soft form factors. We would like to mention here that the hard gluon has virtuality of $m_{B} \Lambda_{\mathrm{QCD}}$, while the energy of the leading light quark scales as $m_{B} / 2$. For the form factors defined in Eq. (2), the factorization formula for the heavy-to-light case at leading order in $1 / m_{B}$ reads [10]

$$
f_{i}\left(q^{2}\right)=C_{i} \xi_{K_{1}}^{a}\left(E_{F}\right)+\Phi_{B} \otimes \mathcal{T}^{\Gamma} \otimes \Phi_{K_{1}},
$$

where $\Phi_{B}$ and $\Phi_{K_{1}}$ are the light-cone distribution amplitudes for the $B$ and $K_{1}$ mesons, respectively. The first term accounts for the soft contributions, with $\xi_{K_{1}}^{a}\left(E_{F}\right)$ representing the soft form factors and the label $a=\perp$, $\|$ corresponding to the perpendicular and parallel polarizations of the $K_{1}$ meson. In Sec. II, we have already expressed the seven form factors in terms of $\xi_{K_{1}}^{\perp, \|}\left(E_{F}\right)$ [cf. Eq. (14)]. The second term in Eq. (22) depicts the hard-spectator scattering contributions. We will see later, while calculating these corrections, that the corresponding amplitude $\mathcal{T}^{\Gamma}$ has endpoint divergences. These end-point divergences are then absorbed in the soft form factors.

\section{A. Vertex renormalization}

Making use of the Passarino-Veltman reduction and keeping the mass term for the $K_{1}$ meson, the vertex diagram shown in Fig. 1 can be evaluated in a systematic way. Let us write $\bar{u}\left(p^{\prime}\right)$ and $u(p)$ as Dirac spinors for light (assumed to be massless) and heavy quarks and introduce a small mass $\lambda$ for the gluon to regulate IR divergences. The UV divergences are analyzed in a naive dimensional regularization (NDR) scheme $(d=4-2 \epsilon)$, and utilizing $\left(\bar{u}\left(p^{\prime}\right) \not p^{\prime}=0, \not p u(p)=m_{b} u(p)\right)$, we get the relation for an arbitrary current $\Gamma$ : 


$$
\begin{aligned}
\bar{u}\left(p^{\prime}\right) \Gamma\left(p^{\prime}, p\right) u(p)= & \frac{\alpha_{s} C_{F}}{4 \pi} \bar{u}\left(p^{\prime}\right)\left[\left\{-\frac{1}{2} \ln \left(\frac{\lambda^{2} m_{b}^{2}}{m_{b}^{2}-q^{2}}\right)-2 \ln \left(\frac{\lambda^{2} m_{b}^{2}}{\left(m_{b}^{2}-q^{2}\right)^{2}}\right)-2 L i_{2}\left(\frac{q^{2}}{m_{b}^{2}}\right)+\frac{m_{B}}{E_{F}} L-3-\frac{\pi^{2}}{2}\right\} \Gamma\right. \\
& +\frac{1}{4}\left\{\frac{1}{\hat{\epsilon}}+3-\ln \left(\frac{m_{b}^{2}}{\mu^{2}}\right)-L^{\prime}\right\} \gamma^{\alpha} \gamma^{\beta} \Gamma \gamma_{\beta} \gamma_{\alpha}+\frac{1}{2 q^{2}}\left\{1-\frac{m_{B}}{2 E_{F}} L\right\} \gamma^{\alpha} \not p \Gamma \not p^{\prime} \gamma_{\alpha} \\
& \left.+\frac{1}{2 q^{2}}\left\{1-L^{\prime}\right\} m_{b} \gamma^{\alpha} \not p \Gamma \gamma_{\alpha}-\frac{1}{2 q^{2}}\left\{2-L^{\prime \prime}\right\} m_{b} \Gamma \not p^{\prime}\right] u(p),
\end{aligned}
$$

where $q^{2}=m_{B}^{2}+m_{K_{1}}^{2}-2 m_{B} E_{F}$. We define the pole $1 / \hat{\epsilon}=1 / \epsilon-\gamma_{E}+\ln 4 \pi$, which in the $\overline{\mathrm{MS}}$ scheme will be subtracted out. The currents are defined in the NDR with an anticommuting $\gamma_{5}$. The remaining quantities are

$$
\begin{aligned}
L & =-\frac{2 E_{F}}{m_{B}-2 E_{F}+\frac{m_{K_{1}}^{2}}{m_{B}}} \ln \left(\frac{2 E_{F}}{m_{B}}-\frac{m_{K_{1}}^{2}}{m_{B}^{2}}\right), \\
L^{\prime} & =L\left(1-\frac{m_{K_{1}}^{2}}{2 E_{F} m_{B}}\right), \\
L^{\prime \prime} & =L\left(4-\frac{m_{B}}{E_{F}}-\frac{2 m_{K_{1}}^{2}}{E_{F} m_{B}}\right) .
\end{aligned}
$$

The coefficient $C_{i}$ at one-loop order is calculated by finding the difference between the full theory and the LEET vertex calculation. It can be seen that all infrared divergent terms in (23) have the same structure as $\Gamma$, so they can be absorbed in the redefinition of the soft form factors $\xi_{K_{1}}^{\perp, \|}$. Introducing a renormalization convention for axial-vector meson form factors that holds exactly to all orders in perturbation theory, we have

$$
A\left(q^{2}\right)=\left(1+\frac{m_{K_{1}}}{m_{B}}\right) \frac{E_{F}}{\Delta} \xi_{K_{1}}^{\perp}\left(E_{F}\right) ; \quad V_{0}\left(q^{2}\right)=\frac{E_{F}}{m_{K_{1}}} \xi_{K_{1}}^{\|}\left(E_{F}\right) .
$$

For a given current $\Gamma$ in Eq. (23), one can find the $\mathcal{O}\left(\alpha_{s}\right)$ corrections by substituting in Eq. (12). Making use of the renormalization convention in Eq. (24) and comparing it with the form factors defined in Eq. (2), we get

$$
\begin{aligned}
A\left(q^{2}\right)= & \left(1+\frac{m_{K_{1}}}{m_{B}}\right) \frac{E_{F}}{\Delta} \xi_{K_{1}}^{\perp}\left(E_{F}\right), \\
V_{0}\left(q^{2}\right)= & \frac{E_{F}}{m_{K_{1}}} \xi_{K_{1}}^{\|}\left(E_{F}\right), \\
V_{1}\left(q^{2}\right)= & \frac{2 E_{F}}{m_{B}+m_{K_{1}}} \xi_{K_{1}}^{\perp}\left(E_{F}\right), \\
V_{2}\left(q^{2}\right)= & \left(1+\frac{m_{K_{1}}}{m_{B}}\right) \frac{E_{F}}{\Delta}\left[\xi_{K_{1}}^{\perp}\left(E_{F}\right)-\left(1+\frac{\alpha_{s} C_{F}}{4 \pi}\left(-2+2 L^{\prime}\right)\right) \xi_{K_{1}}^{\|}\left(E_{F}\right)\right], \\
T_{1}\left(q^{2}\right)= & \frac{E_{F}}{\Delta}\left[\left(1-\frac{m_{K_{1}}^{2}}{m_{B}^{2}}\right)+\frac{\alpha_{s} C_{F}}{4 \pi}\left(-\frac{m_{B}}{E_{F}} L+\ln \left(\frac{m_{b}^{2}}{\mu^{2}}\right)+L^{\prime}\right)\right] \xi_{K_{1}}^{\perp}\left(E_{F}\right), \\
T_{2}\left(q^{2}\right)= & \frac{2 E_{F}}{m_{B}}\left[1+\frac{\alpha_{s} C_{F}}{4 \pi}\left(\frac{m_{B}}{E_{F}} L-\ln \left(\frac{m_{b}^{2}}{\mu^{2}}\right)-L^{\prime}\right)\right] \xi_{K_{1}}^{\perp}\left(E_{F}\right), \\
T_{3}\left(q^{2}\right)= & \left(1+\frac{m_{K_{1}}^{2}}{m_{B}^{2}}\right)\left[\left\{1+\frac{\alpha_{s} C_{F}}{4 \pi}\left(\frac{m_{B}}{E_{F}} L-\ln \left(\frac{m_{b}^{2}}{\mu^{2}}\right)-L^{\prime}\right) \xi_{K_{1}}^{\perp}\left(E_{F}\right)\right\}\right. \\
& \left.-\left\{1+\frac{\alpha_{s} C_{F}}{4 \pi}\left(\frac{3 m_{B}}{2 E_{F}} L-\ln \left(\frac{m_{b}^{2}}{\mu^{2}}\right)+L^{\prime}-2\right) \xi_{K_{1}}^{\|}\left(E_{F}\right)\right\}\right] .
\end{aligned}
$$

Here, one can notice that $V_{1}\left(q^{2}\right)$ does not receive any contribution from the vertex corrections. 


\section{B. Hard-spectator corrections}

The hard-spectator corrections arise at an order $\alpha_{s}$ for which the two-particle light-cone distribution amplitudes of the $B$ and the light $K_{1}$ mesons are given in Appendix A. The momenta of the $b$ quark and the spectator quark before the decay are

$$
p^{\mu}=m_{b} v^{\mu}, \quad l^{\mu}=\frac{l_{+}}{2} n_{+}^{\mu}+l_{\perp}^{\mu}+\frac{l_{-}}{2} n_{-}^{\mu} .
$$

After the quark level transition $b \rightarrow s$ which governs the $B \rightarrow K_{1}$ decay, the momenta of the leading $s$ quark and the corresponding spectator quark in the $K_{1}$ meson are

$$
\begin{aligned}
& k_{1}^{\mu}=u E_{F} n_{-}^{\mu}+k_{\perp}^{\mu}+\left(\frac{\vec{k}_{\perp}^{2}}{4 u E_{F}}+\frac{m_{K_{1}}^{2}}{4 u E_{F}}\right) n_{+}^{\mu}, \\
& k_{2}^{\mu}=\bar{u} E_{F} n_{-}^{\mu}-k_{\perp}^{\mu}+\left(\frac{\vec{k}_{\perp}^{2}}{4 \bar{u} E_{F}}+\frac{m_{K_{1}}^{2}}{4 \bar{u} E_{F}}\right) n_{+}^{\mu},
\end{aligned}
$$

where $\bar{u}=1-u$. All components of $l$ as well as $k_{\perp}$ in $k_{1,2}$ are of the order of $\Lambda_{\mathrm{QCD}}$. It can be seen that $\left(k_{1}+k_{2}\right)^{2} \sim$ $m_{K_{1}}^{2}$ which otherwise scales like $\Lambda_{\mathrm{QCD}}^{2}$. We are interested in hard exchanges where gluon momenta scale as $m_{B} \Lambda_{\mathrm{QCD}}$; therefore, terms proportional to $t h_{-}$will matter.

The contributions to heavy-to-light matrix elements for a given current are given by the convolution formula

$$
\left\langle K_{1}|\bar{q} \Gamma b| B\right\rangle=\frac{4 \pi \alpha_{s} C_{F}}{N_{C}} \int_{0}^{1} d u \int_{0}^{\infty} d l_{+} \mathcal{M}_{j k}^{B} \mathcal{M}_{l i}^{K_{1}} \mathcal{T}_{i j k l}^{\Gamma},
$$

where $\mathcal{M}^{B}, \mathcal{M}^{K_{1}}$ are two-particle light-cone projectors which contain the nonperturbative bound state dynamics. Note that $\mathcal{T}^{\Gamma}$ is the hard scattering amplitude which is calculated from the Feynman diagrams in Fig. 2. The $K_{1}$-meson projector is given as

$$
\mathcal{M}^{K_{1}}=\left[-\frac{i}{4}\left\{f_{K_{1}}^{\perp} \phi^{*} \not p^{\prime} \phi_{\perp}^{K_{1}}(u)+f_{K_{1}}^{\|} \frac{m_{K_{1}}}{E}\left(v \cdot \varepsilon^{*}\right) \phi_{\|}^{K_{1}}(u)\right\} \gamma_{5}\right]_{l i}
$$

with $f_{K_{1}}^{\perp}$ and $f_{K_{1}}^{\|}$denoting the transverse and longitudinal vector meson decay constants and $\phi_{\perp, \|}^{K_{1}}$ denoting the twist-3 two-particle distribution amplitudes (cf. Appendix A).

Similarly the projector for the $B$ meson is

$$
\mathcal{M}^{B}=-\left.\frac{i f_{B} m_{B}}{4}\left[\frac{1+\not p}{2}\left\{\phi_{+}^{B}\left(l_{+}\right) \varkappa_{+}+\phi_{-}^{B}\left(l_{+}\right)\left(\varkappa_{-}-l_{+} \gamma_{\perp}^{v} \frac{\partial}{\partial l_{\perp}^{v}}\right)\right\} \gamma_{5}\right]_{j k}\right|_{l=\left(l_{+} / 2\right) n_{+}}
$$

where $f_{B}$ is the $B$-meson decay constant. Last but not least, the hard scattering amplitude from Fig. 2 is

$$
\mathcal{T}_{i j k l}^{\Gamma}=\left[\Gamma \frac{m_{b}(1+\not p)+\not l-\not k_{2}}{\left(m_{b} v+l-k_{2}\right)^{2}-m_{b}^{2}} \gamma_{\mu}+\gamma_{\mu} \frac{\not k_{1}+\not k_{2}-\not l}{\left(k_{1}+k_{2}-l\right)^{2}} \Gamma\right]_{i j} \frac{1}{\left(l-k_{2}\right)^{2}}\left[\gamma^{\mu}\right]_{k l} .
$$

Working in the context of the LEET, we are interested only in the hard gluon exchange. The gluon propagator with momenta defined in Eqs. (26)-(28), when expanded, gives $\left(l-k_{2}\right)^{2} \sim-2 l_{+} \bar{u} E_{F}$ which scales as $m_{B} \Lambda_{\mathrm{QCD}}$. The first term in Eq. (32) has the numerator $l-\not k_{2} \sim-\bar{u} E_{F} \not h_{-}$, which is the surviving term at the scale $m_{B} \Lambda_{\mathrm{QCD}}$. When all the dust settles, the contributions of hard exchanges after neglecting terms of order $\Lambda_{\mathrm{QCD}} / m_{B}$ are

$\mathcal{T}_{i j k l}^{\Gamma} \simeq\left[\Gamma \frac{m_{b}(1+\not h)-\bar{u} E_{F} \not h_{-}}{4 \bar{u}^{2} l_{+} m_{b} E_{F}^{2}} \gamma_{\mu}+\gamma_{\mu} \frac{E_{F} \not h_{-}-l}{4 \bar{u} l_{+}^{2} E_{F}^{2}} \Gamma\right]_{i j}\left[\gamma^{\mu}\right]_{k l}$.

The term $m_{b}(1+\not p)$ diverges logarithmically for $\bar{u} \rightarrow 0$ as the function $\phi(u)$ vanishes only linearly in the leading twist. These so-called end-point divergences can be absorbed in soft form factors in our factorization scheme (22) as they do not break heavy or large recoil symmetry relations. This can be easily verified by looking at the similarity of its current structure with the one defined in Eq. (12). In this study, we can go beyond twist-2 and work with the twist-3 distribution amplitudes. Now the twist-3 distributions are suppressed by $1 / m_{B}$ which are compensated by the linear term $m_{B} / \Lambda_{\mathrm{QCD}}$ in $\mathcal{M}^{K_{1}}$ for the case $\bar{u} \rightarrow 0$. Therefore, such terms contribute at leading order in soft factors $\xi_{K_{1}}^{\perp, \|}$. A similar argument can be made for the second term in Eq. (33) where the twist-3 contributions are factored in soft form factors for the case $\bar{u} \rightarrow 0$ as end-point divergences. In short, all the twist-3 contributions preserve heavy-quark-large-energy symmetries. There remains just one term-i.e., $-\bar{u} E_{F} \not h_{-}$in the first numerator of Eq. (33) - that breaks the symmetry. This term is linear in $\bar{u}$, and after evaluating the convolution formula in Eq. (29), terms proportional to $\phi_{+}^{B}\left(l_{+}\right)$survive. 
Now, let us focus on $\phi_{-}^{B}\left(l_{-}\right)$in $\mathcal{M}^{B}$ given in Eq. (31). The $h_{-}$from the term $\left(-\bar{u} E_{F} \not h_{-}\right)$, when multiplied by $\phi_{-}^{B}\left(l_{-}\right)\left(\not_{-}-l_{+} \gamma_{\perp}^{\nu} \frac{\partial}{\partial l_{\perp}^{\nu}}\right)$, gives a term proportional to $l_{+} \gamma_{\perp}$ as $h_{-} h_{-}=0$. This remaining term involving $\phi_{-}^{B}\left(l_{-}\right)$can preserve heavy-quark-large-energy symmetry.

Just to give some details on how the symmetry breaking correction terms appear in the form factors, the detailed calculation of the hard-spectator corrections to the form factor $V_{2}\left(q^{2}\right)$ is given in Appendix C. A similar procedure is adopted for the form factors $T_{1,2,3}\left(q^{2}\right)$. Now using different possible Dirac gamma structures in Eq. (29) and comparing with Eq. (2), the complete results of the form factors after including vertex and hard-spectator corrections become

$$
\begin{gathered}
A\left(q^{2}\right)=\left(1+\frac{m_{K_{1}}}{m_{B}}\right) \frac{E_{F}}{\Delta} \xi_{K_{1}}^{\perp}\left(q^{2}\right), \\
V_{0}\left(q^{2}\right)=\frac{E_{F}}{m_{K_{1}}} \xi_{K_{1}}\left(q^{2}\right), \\
V_{1}\left(q^{2}\right)=\frac{2 E_{F}}{m_{B}+m_{K_{1}}} \xi_{K_{1}}^{\perp}\left(q^{2}\right), \\
V_{2}\left(q^{2}\right)=\left(1+\frac{m_{K_{1}}}{m_{B}}\right) \frac{E_{F}}{\Delta}\left[\xi_{K_{1}}^{\perp}\left(q^{2}\right)-\left\{1+\frac{\alpha_{s} C_{F}}{4 \pi}\left(-2+2 L^{\prime}-\frac{q^{2}}{m_{B}^{2}-m_{K_{1}}^{2}} \frac{m_{B}}{2 E_{F}} \frac{\Delta F_{\|}}{\xi^{\|}\left(q^{2}\right)}\right)\right\} \xi_{K_{1}}\left(q^{2}\right)\right], \\
T_{2}\left(q^{2}\right)=\frac{E_{F}}{\Delta}\left[\left(1-\frac{m_{K_{1}}^{2}}{m_{B}^{2}}\right)+\frac{\alpha_{s} C_{F}}{4 \pi}\left\{-\frac{m_{B}}{E_{F}} L+\ln \left(\frac{m_{b}^{2}}{\mu^{2}}\right)+L^{\prime}+\frac{\Delta m_{B}}{4 E_{F}^{2}} \frac{\Delta F_{\perp}}{\xi_{K_{1}}^{\perp}}\left(q^{2}\right)\right\}\right] \xi_{K_{1}}^{\perp}\left(q^{2}\right), \\
\left.\left.\left.T_{3}\left(q^{2}\right)=\left(1-\frac{\alpha_{s} C_{F}}{4 \pi}\left\{\frac{-m_{B}}{E_{F}} L+\ln \left(\frac{m_{b}^{2}}{m^{2}}\right)+L^{\prime}\right)+\frac{m_{B}}{4 E_{F}} \frac{\Delta F_{\perp}}{\xi_{K_{1}}^{\perp}}\right)\right] q^{2}\right)\right\} \xi_{K_{1}}^{\perp}\left(q^{2}\right), \\
\left.\left.\left.-\left\{1+\frac{\alpha_{s} C_{F}}{4 \pi}\left(\frac{m_{B}}{E_{F}} L-\ln \left(\frac{m_{b}^{2}}{\mu^{2}}\right)-L^{\prime}-\frac{m_{B}^{2}}{m_{B}^{2}-m_{K_{1}}^{2}} \frac{m_{B}}{4 E_{F}} \frac{\Delta m_{\perp} L}{2 E_{F}}-\ln \left(\frac{m_{b}^{2}}{\mu^{2}}\right)+L^{\prime}-2\right\} q^{2}\right)\right\} \xi_{K_{1}}^{\perp}\left(q^{2}\right)\right)\right],
\end{gathered}
$$

where the quantities $\Delta F_{\perp}$ and $\Delta F_{\|}$can be written as

$$
\begin{aligned}
\Delta F^{\perp} & =\frac{8 \pi^{2} f_{B} f_{K_{1}}^{\perp}}{N_{C} m_{B}}\left\langle l_{+}^{-1}\right\rangle_{+}\left\langle\bar{u}^{-1}\right\rangle^{\perp}, \\
\Delta F^{\|} & =\frac{8 \pi^{2} f_{B} f_{K_{1}}^{\|}}{N_{C} m_{B}}\left\langle l_{+}^{-1}\right\rangle_{+}\left\langle\bar{u}^{-1}\right\rangle^{\|} .
\end{aligned}
$$

The leading twist moments given for the $K_{1}$ and $B$ mesons are

$$
\left\langle\bar{u}^{-1}\right\rangle^{\perp, \|}=\int d u \frac{\phi_{\perp, \|}^{K_{1}}(u)}{\bar{u}}
$$

and

$$
\left\langle l_{+}^{-1}\right\rangle_{+}=\int d l_{+} \frac{\phi_{+}^{B}\left(l_{+}\right)}{l_{+}} .
$$

Again, we can see that $V_{1}\left(q^{2}\right)$ does not receive any symmetry breaking correction term, i.e., neither from the vertex nor from hard-spectator corrections.

\section{NUMERICAL ANALYSIS AND APPLICATIONS}

\section{A. Form factor analysis}

In this section, we perform the numerical analysis of the form factors. To calculate the $\alpha_{s}$ corrections to the form factors, we use the $B$-meson decay constant, $f_{B}=0.195 \pm$ $0.01 \mathrm{GeV}$. We already mentioned in Eq. (1) that the physical states $K_{1}(1200,1400)$ are the mixture of flavor states $K_{1(A, B)}$; therefore, the decay constant corresponding to physical states $f_{K_{1}}^{\perp}\left(f_{K_{1}}^{\|}\right)$can be obtained by mixing $f_{K_{1(A, B)}}^{\perp}=0.122,0.0884\left(f_{K_{1(A, B)}}^{\|}=0.17,0.125\right) \mathrm{GeV} \quad$ [26]. All quantities in hard scattering amplitudes are calculated at the scale of $1.5 \mathrm{GeV}$. For the flavor states, we expand $\phi_{K_{1}}^{\perp, \|}$ up to the second Gegenbauer moment [26]: 


$$
\begin{aligned}
& \phi_{K_{1 A}}^{\perp}=6 u \bar{u}\left(a_{0 A}^{\perp}+3 a_{1 A}^{\perp}(2 u-1)+\frac{3}{2} a_{2 A}^{\perp}\left(5\left((2 u-1)^{2}-1\right)\right),\right. \\
& \phi_{K_{1 B}}^{\perp}=6 u \bar{u}\left(1+3 a_{1 B}^{\perp}(2 u-1)+\frac{3}{2} a_{2 B}^{\perp}\left(5\left((2 u-1)^{2}-1\right)\right),\right. \\
& \phi_{K_{1} A}^{\|}=6 u \bar{u}\left(1+3 a_{1 B}^{\|}(2 u-1)+\frac{3}{2} a_{2 B}^{\|}\left(5\left((2 u-1)^{2}-1\right)\right),\right. \\
& \phi_{K_{1} B}^{\|}=6 u \bar{u}\left(a_{0 B}^{\|}+3 a_{1 B}^{\|}(2 u-1)+\frac{3}{2} a_{2 A}^{\|}\left(5\left((2 u-1)^{2}-1\right)\right),\right.
\end{aligned}
$$

and the values of $a_{0,1,2}^{\perp, \|}$ are given in [26]. In the case of soft form factors, we need the numerical values of the functions $\xi_{K_{1}}^{\|}, \xi_{K_{1}}^{\perp}$. For this, we parametrize them in terms of the energy in the large recoil limit as

$$
\left(\frac{m_{B}+m_{K_{1}}}{m_{B}} \frac{E_{F}}{\Delta} \xi_{K_{1}}^{\perp}, \frac{E_{F}}{m_{K_{1}}} \xi_{K_{1}}^{\|}\right)=\left\{A(0), V_{0}(0)\right\} \times\left(\frac{m_{B}}{2 E_{F}}\right)^{2}
$$

where we use the LCSR form factor values $\left(A_{K_{1 A}}(0)=\right.$ $\left.0.45 \pm 0.09, A_{K_{1 B}}(0)=-0.37_{-0.06}^{+0.10}\right) \quad$ and $\quad\left(V_{0\left(K_{1 A}\right)}(0)=\right.$ $\left.0.22 \pm 0.04, V_{0\left(K_{1 B}\right)}(0)=-0.45_{-0.08}^{+0.12}\right)$ [20]. The values of the other form factors for these flavor states are calculated in [20] and are summarized in Table IV of Ref. [21]. It is worth mentioning that the values of the form factors calculated in [20] do not take into account gluon radiative corrections. Also, we emphasize here that the form factors of the physical mass states $K_{1}(1270,1400)$ also mix, i.e.,

$$
\begin{aligned}
& \mathcal{A}_{i}^{(1270)}(0)=\mathcal{A}_{i}^{K_{1 A}}(0) \sin \theta_{K_{1}}+\mathcal{A}_{i}^{K_{1 B}}(0) \cos \theta_{K_{1}} \\
& \mathcal{A}_{i}^{(1400)}(0)=\mathcal{A}_{i}^{K_{1 A}}(0) \cos \theta_{K_{1}}-\mathcal{A}_{i}^{K_{1 B}}(0) \sin \theta_{K_{1}},
\end{aligned}
$$

where $\mathcal{A}_{i}(0)$ can be $A(0), V_{0,1,2}(0)$, and $T_{1,2,3}(0)$. The form factors $A_{0}\left(q^{2}\right)$ and $V_{0}\left(q^{2}\right)$ correspond to our renormalization convention to denote perpendicular $\left(\xi_{K_{1}}^{\perp}\right)$ and parallel $\left(\xi_{K_{1}}^{\|}\right)$components, respectively, and $V_{1}\left(q^{2}\right)$ does not receive any radiative corrections. The rest of the form factors $V_{2}\left(q^{2}\right), T_{1,2,3}\left(q^{2}\right)$ have contributions from the symmetry breaking terms.

Using these numerical values of different input parameters at $\alpha_{s}=0.34$ and $\mu=1.47 \mathrm{GeV}$, the form factors against momentum transfer $q^{2}$ are plotted in Fig. 3. As an input we used the values of the form factors calculated in [20], where it can be seen that their values for the states $K_{1 A, 1 B}$ at $q^{2}=0$ given in Table IV of [21] are prone to uncertainties. Using these input values along with the other parameters, the form factors extrapolated with $q^{2}$ using Eq. (45) are plotted in Fig. 3. In these plots, the black band corresponds to the uncertainties in the LCSR form factors without symmetry breaking corrections. The blue band corresponds to the same form factors and uncertainties but this time including the symmetry breaking corrections calculated here. We consider that the tensor form factors are renormalized at $\mu=m_{b}$. In Fig. 3 we can see that in most of the $q^{2}$ region, the two bands overlap significantly, showing that the symmetry breaking corrections are masked by the uncertainties inherited through the input values of the form factors and other parameters. The most prominent effects in almost all the $q^{2}$ range come in the tensor form factors $T_{2}\left(q^{2}\right)$ and $T_{3}\left(q^{2}\right)$. Quantitatively, we can see that the symmetry breaking corrections change the LEET form factors $V_{2}\left(q^{2}\right)$ and $T_{1,2,3}\left(q^{2}\right)$ by less than $10 \%$. It is worth mentioning that the major uncertainty lies in hard-spectator corrections due to LCDA of the $B$ meson. In the past, due to nonavailability of constraints on $\lambda_{B}$, this uncertainty could rise as high as $\pm 50 \%$ [10]. This uncertainty was constrained by the $B A B A R$ analysis of $B \rightarrow \gamma l \nu$ [27] at small recoil. This can be further improved by a similar analysis by $B A B A R$ for large recoil radiative decay. The BABAR experiment has an upper limit of $\lambda_{B} \sim$ $669 \mathrm{MeV}$ and a lower limit of $\lambda_{B} \sim 300 \mathrm{MeV}$. Their analysis was further improved in Ref. [28], as the former does not consider highly energetic photons and radiative or power corrections. For our calculations of form factors for semileptonic decay, the value of $\lambda_{B} \sim 0.35 \mathrm{GeV}$ seems to be optimal. In the context of the study [28], we expect more uncertainty at large recoil than at small recoil. This is the reason why radiative corrections become important for precision calculation, especially in the regime of $q^{2} \sim$ $1-3 \mathrm{GeV}^{2}$ where the symmetry breaking overlaps less significantly with uncertainties only for the form factors $T_{2,3}\left(q^{2}\right)$. The constraint on the $B$-meson light-cone distribution amplitude, along with the uncertainty in the $K_{1}$ decay constant, makes our results susceptible to $\pm 25 \%$ uncertainty.

\section{B. Applications}

To see how the symmetry breaking corrections influence the values of the observables, we study the implications of the modified form factors on the zero position of the forward-backward asymmetry and the longitudinal lepton polarization asymmetry $\left(P_{L}\right)$ for the decay channel $B \rightarrow K_{1} \mu^{+} \mu^{-}$. 

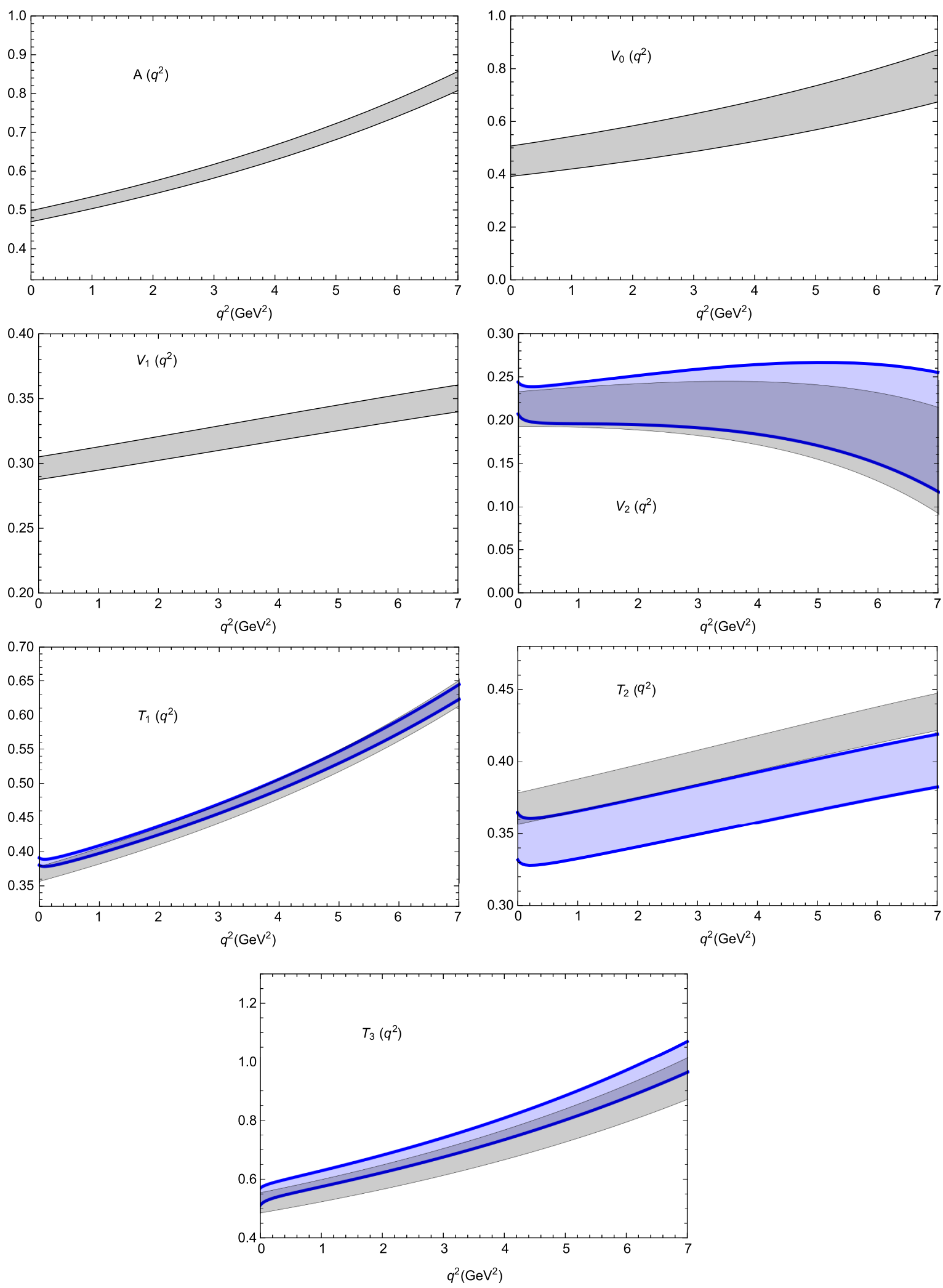

FIG. 3. Form factors are plotted with $q^{2}$. Using the uncertainties in the form factors calculated in $[20,21]$ at $q^{2}=0$ along with the other input parameters, and by parametrizing them with $q^{2}$ through Eq. (45), their trend without symmetry breaking corrections is shown by the black band. The blue band corresponds to the same uncertainties in form factor but this time including symmetry breaking corrections. The hard corrections are calculated at $\alpha_{s}=0.34$ at the scale $\mu=1.47 \mathrm{GeV}$. Tensor form factors are renormalized at the $b$-quark mass, i.e., $\mu=m_{b}$. 


\section{Forward-backward asymmetry}

The forward-backward asymmetry and its zero position in $B \rightarrow K_{1} \ell^{+} \ell^{-}$provide an interesting tool to look for the physics beyond the SM. At leading order in the SM, this asymmetry has very weak dependence on the form factors. Therefore, it is interesting to see the effects of the form factors incorporating the symmetry breaking corrections on the behavior and the zero position of the forward-backward asymmetry. The effective Hamiltonian responsible for the decay under consideration is

$$
\mathcal{H}=\frac{G_{F}}{\sqrt{2}} V_{t s}^{*} V_{t b} \sum_{i=1}^{10} C_{i}(\mu) \mathcal{O}_{i}(\mu),
$$

where $\mathcal{O}_{i}$ are four-quark local operators and $C_{i}$ are Wilson coefficients calculated in the naive dimensional regularization (NDR) scheme at a scale $\mu$. At the quark level, the corresponding amplitude for the underlying transition $b \rightarrow s \ell^{+} \ell^{-}$is

$$
\begin{aligned}
\mathcal{M}\left(b \rightarrow s \ell^{+} \ell^{-}\right)= & \frac{G_{F} \alpha}{\sqrt{2}} V_{t s}^{*} V_{t b}\left[C_{9}^{\mathrm{eff}}\left(\bar{s} \gamma_{\mu} L b\right)\left(\bar{\ell} \gamma^{\mu} \ell\right)\right. \\
& +C_{10}\left(\bar{s} \gamma_{\mu} L b\right)\left(\bar{\ell} \gamma^{\mu} \gamma^{5} \ell\right) \\
& \left.-2 \frac{m_{b}}{q^{2}} C_{7}^{\mathrm{eff}}\left(\bar{s} i \sigma_{\mu \nu} \frac{q^{\nu}}{q^{2}} R b\right)\left(\bar{\ell} \gamma^{\mu} \ell\right)\right]
\end{aligned}
$$

where $L=\frac{1-\gamma_{5}}{2}, R=\frac{1+\gamma_{5}}{2}$, with $m_{b}$ the mass of the $b$ quark and $C_{7}^{\text {eff }}=C_{7}-C_{5} / 3-C_{6}$. Here, $C_{9}^{\text {eff }}$ contains both short-distance and long-distance contributions, given by

$$
C_{9}^{\text {eff }}\left(q^{2}\right)=C_{9}(\mu)+Y_{\text {pert }}(\hat{s})+Y_{\mathrm{LD}}\left(q^{2}\right)
$$

where $\hat{s}=\frac{q^{2}}{m_{b}^{2}}$. Note that $Y_{\text {pert }}$ represents the perturbative contributions, and $Y_{\mathrm{LD}}$ is the long-distance part. The $Y_{\text {pert }}$ is given in [29]:

$$
\begin{aligned}
Y_{\text {pert }}= & h\left(\hat{m}_{c}, \hat{s}\right) C_{0}-\frac{1}{2} h(1, \hat{s})\left(4 C_{3}+4 C_{4}+3 C_{5}+C_{6}\right) \\
& -\frac{1}{2} h(0, \hat{s})\left(C_{3}+3 C_{4}\right)+\frac{2}{9}\left(3 C_{3}+C_{4}+3 C_{5}+C_{6}\right) .
\end{aligned}
$$

As in the LEET, we are working below the $J / \psi$ resonances, i.e., dilepton invariant mass of up to $q^{2}=7 \mathrm{GeV}$; therefore, we will ignore the contribution from $Y_{\mathrm{LD}}$. The study of the branching fraction and asymmetries in the decay under consideration is a bit complicated due to the mixing of $K_{1 A}$ and $K_{1 B}$ states, as already pointed out in Eq. (1).

The amplitude of the decay $B \rightarrow K_{1} \mu^{+} \mu^{-}$is found by sandwiching the different $\left(\bar{s} \Gamma_{\mu} b\right)$ currents between the $B$ and $K_{1}$ mesons and expressing them in terms of form factors as given in Eq. (2). Using the standard procedure, the corresponding partial decay width can be given as

$$
\begin{aligned}
d \Gamma & \left(B \rightarrow K_{1} \mu^{+} \mu^{-}\right) \\
& =\frac{\sqrt{\lambda}}{1024 \pi^{4} m_{B}^{3}} d \cos \theta d q^{2}\left|\mathcal{M}\left(B \rightarrow K_{1} \mu^{+} \mu^{-}\right)\right|^{2},
\end{aligned}
$$

where $\theta$ is the angle between the $B$ meson and $\mu^{+}$. The quantity $\lambda$ is given as [13]

$$
\begin{aligned}
\lambda & \equiv \lambda\left(q^{2}, m_{B}^{2}, m_{K_{1}}^{2}\right) \\
& =\left[\left(1-\frac{q^{2}}{m_{B}^{2}}\right)^{2}-\frac{2 m_{K_{1}}^{2}}{m_{B}^{2}}\left(1-\frac{q^{2}}{m_{B}^{2}}\right)+\frac{m_{K_{1}}^{4}}{m_{B}^{4}}\right]^{1 / 2} .
\end{aligned}
$$

The differential forward-backward asymmetry is defined as

$\frac{d \mathcal{A}_{F B}}{d q^{2}}=\int_{0}^{1} d(\cos \theta) \frac{d^{2} \Gamma}{d q^{2} d \cos \theta}-\int_{-1}^{0} d(\cos \theta) \frac{d^{2} \Gamma}{d q^{2} d \cos \theta}$.

The differential decay width can be calculated by expressing the matrix elements of $\left\langle K_{1}\left|\left(\bar{s} \Gamma_{\mu} b\right)\right| B\right\rangle$ using quark level currents given in Eq. (48), in which the hadronic part is parametrized in terms of the form factors. Consequently, the normalized differential forward-backward asymmetry takes the form

$$
\begin{aligned}
\frac{d \mathcal{A}_{F B}}{d q^{2}}= & -\frac{1}{d \Gamma / d q^{2}} \frac{G_{F}^{2}\left|V_{t s}^{*} V_{t b}\right|^{2}}{128 \pi^{3}} m_{B}^{3} \lambda\left(q^{2}, m_{K_{1}}^{2}\right)^{2}\left(\frac{\alpha}{4 \pi}\right)^{2} \frac{8 q^{2}}{m_{B}^{2}} C_{10} V_{1}\left(q^{2}\right) A\left(q^{2}\right) \\
& \times \Re\left[C_{9}^{\mathrm{eff}}+\frac{m_{b}}{q^{2}} C_{7}^{\mathrm{eff}}\left(\left(m_{B}+m_{K_{1}}\right) \frac{T_{1}\left(q^{2}\right)}{A\left(q^{2}\right)}+\left(m_{B}-m_{K_{1}}\right) \frac{T_{2}\left(q^{2}\right)}{V_{1}\left(q^{2}\right)}\right)\right] .
\end{aligned}
$$

The expression of $\mathcal{A}_{F B}$ given in Eq. (54) involves the ratio of the form factors $\frac{T_{1}\left(q^{2}\right)}{A\left(q^{2}\right)}$ and $\frac{T_{2}\left(q^{2}\right)}{V_{1}\left(q^{2}\right)}$. From Eqs. (34)-(40) we can see that the symmetry breaking corrections appear in the form factors $T_{1}\left(q^{2}\right)$ and $T_{2}\left(q^{2}\right)$. Hence, we can expect the deviation in the numerical values of both the amplitude and zero position of $\mathcal{A}_{F B}$ from the HQET form factors, and Fig. 4 depicts this fact. Notice that the shift of the zero position of $\mathcal{A}_{F B}$ is about $10 \%$, indicating that before attributing any deviation in the zero position of the $\mathcal{A}_{F B}$ as a NP, it is important to take into account that the shift arises due to the symmetry breaking corrections in the form factors. 


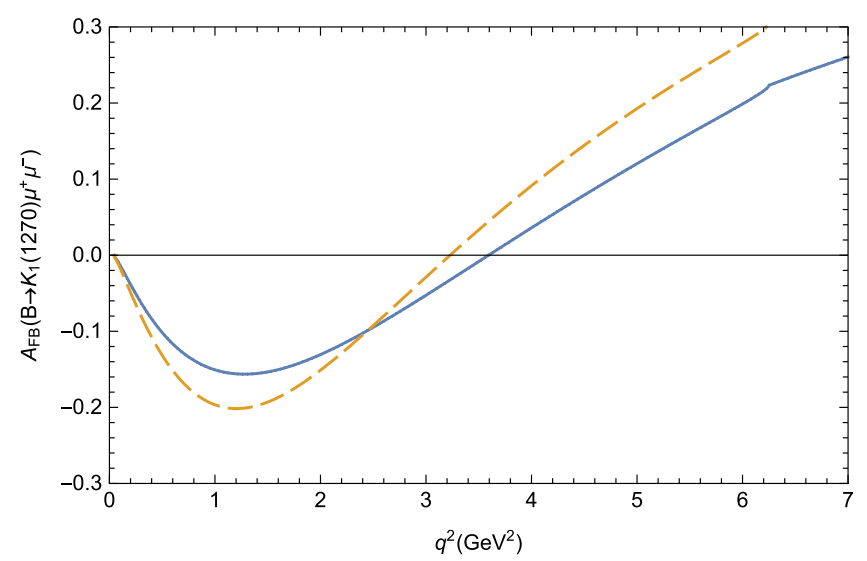

FIG. 4. Forward-backward asymmetry as a function of $q^{2}$. The solid and dashed lines correspond to the form factors without and with corrections, respectively.

\section{Longitudinal lepton polarization}

In principle, many angular observables can be conceived; however, we are interested in longitudinal lepton polarization of the lepton pair. Since we obtain the lepton pair from either the off-shell photon, the $Z$ boson or some other neutral vector boson, the vertex of the decay to the lepton pair has Lorentz structure of either $(V-A)$ or $(V+A)$. Therefore, we can assign a different combination of possible helicities, and they are summarized in Appendix B. The decay amplitude in terms of the lepton and hadron helicity amplitudes can be written as [30]

$$
\begin{aligned}
\mathcal{M} & \left(B \rightarrow K_{1} \mu^{+} \mu^{-}\right) \\
& =-\sum_{i}(\mathcal{L}(L, i) \mathcal{H}(L, i)-\mathcal{L}(R, i) \mathcal{H}(R, i)),
\end{aligned}
$$

where $\mathcal{L}(L)=\bar{\mu} \gamma_{\mu}\left(1-\gamma_{5}\right) \mu$ and $\mathcal{L}(R)=\bar{\mu} \gamma_{\mu}\left(1+\gamma_{5}\right) \mu$ are the lepton pair currents. After integrating out $\theta$ and $\phi$, which are defined in the rest frame of the lepton pair (cf. Appendix B), we get the following result:

$$
\frac{d \Gamma_{i}}{d q^{2}}=\frac{\sqrt{\lambda}}{96 \pi^{3} m_{B}^{3}}\left[|\mathcal{H}(L, i)|^{2}+|\mathcal{H}(R, i)|^{2}\right]
$$

where $\mathcal{H}(L, i), \mathcal{H}(R, i)$ are the hadronic transition amplitudes, which are summarized in Appendix B. The asymmetry in longitudinal-lepton polarization is written as

$$
\begin{aligned}
P_{L}= & \int_{0}^{1} d \cos \theta_{1}\left(|\mathcal{L}(L, 0) \mathcal{H}(L, 0)|^{2}\right) \\
& -\int_{-1}^{0} d \cos \theta_{1}\left(|\mathcal{L}(R, 0) \mathcal{H}(R, 0)|^{2}\right),
\end{aligned}
$$

where $|\mathcal{L}(R, 0)|^{2}=|\mathcal{L}(R, 0)|^{2}=4 q^{2} \sin ^{2} \theta_{1}$. Integrating $\theta_{1}$ in Eq. (57) and normalizing it with the full differential decay rate in the denominator, one gets

$$
\bar{P}_{L}=\frac{|\mathcal{H}(L, 0)|^{2}-|\mathcal{H}(R, 0)|^{2}}{|\mathcal{H}(L, i)|^{2}+|\mathcal{H}(R, i)|^{2}}
$$

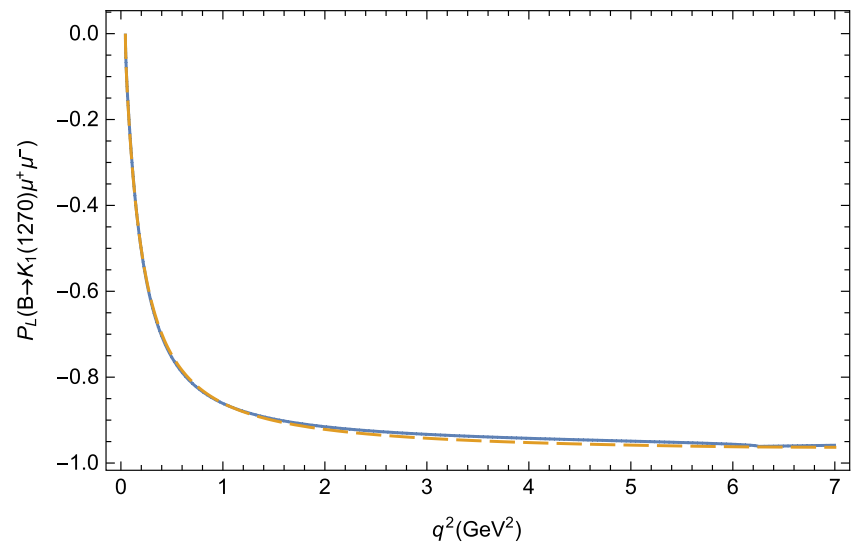

FIG. 5. Longitudinal lepton polarization asymmetry due to symmetry breaking corrections. The description of the solid and dashed lines is the same as in Fig. 4.

Upon substituting the expressions of $\mathcal{H}(L, i)$ and $\mathcal{H}(R, i)$, the result for the lepton-polarization asymmetry reads

$$
\begin{aligned}
P_{L}= & -4 C_{9}^{\text {eff }} C_{10} \mathcal{C}^{\prime 2}\left|V_{1}\left(q^{2}\right)\right|^{2}-4 C_{9}^{\text {eff }} C_{10} \mathcal{D}^{\prime 2}\left|V_{2}\left(q^{2}\right)\right|^{2} \\
& +4 C_{9}^{\text {eff }} C_{10} \mathcal{C}^{\prime} \mathcal{D}^{\prime} V_{1}\left(q^{2}\right) V_{2}\left(q^{2}\right) \\
& -4 C_{7} C_{10} \mathcal{B}^{\prime} \mathcal{C}^{\prime} T_{2}\left(q^{2}\right) V_{1}\left(q^{2}\right) \\
& -4 C_{7} C_{10} \mathcal{A}^{\prime} \mathcal{D}^{\prime} T_{3}\left(q^{2}\right) V_{2}\left(q^{2}\right) \\
& +4 C_{7} C_{10} \mathcal{B}^{\prime} \mathcal{D}^{\prime} T_{2}\left(q^{2}\right) V_{2}\left(q^{2}\right)
\end{aligned}
$$

where the quantities $\mathcal{A}^{\prime}, \mathcal{B}^{\prime}, \mathcal{C}^{\prime}, \mathcal{D}^{\prime}$ are given as

$$
\begin{aligned}
\mathcal{A}^{\prime} & =\frac{\lambda}{m_{B}^{2}-m_{K_{1}}^{2}}, \quad \mathcal{B}^{\prime}=3 m_{K_{1}}^{2}+m_{B}^{2}-q^{2}, \\
\mathcal{C}^{\prime} & =\left(m_{B}-m_{K_{1}}\right)\left(m_{K_{1}}^{2}-m_{B}^{2}+q^{2}\right), \quad \mathcal{D}^{\prime}=\frac{\lambda}{m_{B}-m_{K_{1}}} .
\end{aligned}
$$

Now using the form factors from Eqs. (34)-(40), we get $P_{L}$ in terms of two soft form factors $\xi_{K_{1}}^{\perp, \|}$ and hard spectator factors $\Delta F_{\perp, \|}$. The behavior of $P_{L}$ as a function of dilepton mass squared is shown in Fig. 5. As can be observed, there is no difference in the value after incorporating the symmetry breaking corrections to the form factors for this particular observable. Therefore, any significant deviation from the SM prediction of this physical observable in the $B \rightarrow K_{1} \ell^{+} \ell^{-}$decay will be a hint of new physics.

\section{CONCLUSION}

In this work, radiative corrections to form factors at oneloop order are calculated in the $B \rightarrow K_{1} \mu^{+} \mu^{-}$decay. These corrections are significant at large recoil $q^{2} \sim 1-7 \mathrm{GeV}^{2}$ for heavy-to-light transitions. We employed a factorization scheme in the context of the LEET to take into account the soft- and hard-gluon exchanges. The vertex corrections 
are found by matching effective theory with full theory at the one-loop level. These corrections do not break symmetry relations and appear as $\alpha_{s}$ corrections in the form factors [cf. Eq. (25)]. The hard-spectator corrections break symmetry relations, and these are calculated via light-cone distribution amplitudes. We found that the accumulated corrections to form factor relations shift the zero position of the forward-backward asymmetry by $10 \%$. Therefore, we can say that the symmetry breaking corrections, if not calculated, would somehow mix with the possible NP for this observable in the $B \rightarrow K_{1} \mu^{+} \mu^{-}$decay. Contrary to the forward-backward asymmetry, the longitudinal lepton polarization asymmetry is hardly affected by these symmetry breaking corrections. Therefore, any significant difference, especially in the longitudinal lepton polarization asymmetry, if observed experimentally, would be indicative of some physics beyond the SM.

\section{ACKNOWLEDGMENTS}

M. J. A. would like to thank Professor Andrzej Czarnecki for the opportunity to work as a visiting professor in his group at the University of Alberta during a sabbatical leave from Quaid-i-Azam University. The work of M. J. A. is partly supported by the Natural Sciences and Engineering Research Council of Canada.

\section{APPENDIX A: PARTON DISTRIBUTION AMPLITUDES}

\section{1. $B$-meson parton distribution amplitude}

The two-particle light-cone matrix element with $B$ momentum $m_{B} v$ and two functions $\phi_{ \pm}^{B}(t)$ in coordinate space compatible with Lorentz decomposition is [8]

$$
\begin{aligned}
M(z) & \equiv\left\langle 0\left|\bar{q}_{\beta}(z) P(z, 0) q_{\alpha}(0)\right| \bar{B}(p)\right\rangle \\
& =-\frac{i f_{B} m_{B}}{4}\left[\frac{1+\not p}{2}\left\{2 \phi_{+}^{B}(t)+\frac{\phi_{-}^{B}(t)-\phi_{+}^{B}(t)}{t} \not t\right\} \gamma_{5}\right]_{\alpha \beta} .
\end{aligned}
$$

The factor $-\frac{i f_{B} m_{B}}{4}$ is chosen according to the normalization of the pseudoscalar meson, i.e., $\left\langle 0\left|\bar{q}_{\beta}\left[\gamma_{5}\right]_{\beta \alpha} q_{\alpha}\right| \bar{B}(p)\right\rangle$ and $t=v \cdot z$. The path ordered exponential in Eq. (A1) is given as

$$
P\left(z_{2}, z_{1}\right)=P \exp \left(i g_{s} \int_{z_{2}}^{z_{1}} d z^{\mu} A_{\mu}(z)\right) .
$$

Finding the momentum space projector $M^{B}$ of $M(z)$,

$$
\begin{aligned}
\int d^{4} z M(z) A(z) & =\int \frac{d^{4} l}{(2 \pi)^{4}} A(l) \int d^{4} z e^{-i l z} M(z), \\
& =\left.\int_{0}^{\infty} d l_{+} M^{B} A(l)\right|_{l=\left(l_{+} / 2\right) n_{+}},
\end{aligned}
$$

where $A(z)$ is the hard scattering amplitude in coordinate space and $A(l)$ is its momentum representation. Now, being consistent with our definition of $l$, i.e.,

$$
l^{\mu}=\frac{l_{+}}{2} n_{+}^{\mu}+\frac{l_{-}}{2} n_{-}^{\mu}+l_{\perp}^{\mu}
$$

the coordinate function $\phi_{ \pm}^{B}(t)$ in momentum space is

$$
\phi_{ \pm}^{B}(t) \equiv \int_{0}^{\infty} d \omega e^{-i \omega t} \phi_{ \pm}^{B}(\omega)
$$

In the heavy-quark limit, the hard scattering amplitude $A(l)$ in the light meson in the $n_{-}$direction is independent of $l_{-}$. So $A(l)=A^{0}\left(l_{+}\right)+l_{\perp}^{\mu} A_{\mu}^{1}\left(l_{+}\right)+O\left(1 / m_{B}\right)$. Moreover, the derivative is given after dropping the $l_{-}$term:

$$
\frac{\partial}{\partial l_{\mu}}=n_{-}^{\mu} \frac{\partial}{\partial l_{\mu}}+\frac{\partial}{\partial l_{\perp \mu}} .
$$

Substituting Eqs. (A5) and (A6) along with $A(l)$ in Eq. (A3), we find

$$
M_{\beta \alpha}^{B}=-\left.\frac{i f_{B} m_{B}}{4}\left[\frac{1+\not p}{2}\left\{\phi_{+}^{B}(\omega) h_{+}+\phi_{-}^{B}(\omega)\left(\not h_{-}-l_{+} \gamma_{\perp}^{\nu} \frac{\partial}{\partial l_{\perp}^{\nu}}\right)\right\} \gamma_{5}\right]_{\beta \alpha}\right|_{l=\left(l_{+} / 2\right) n_{+}} .
$$

\section{2. $K_{1}$-meson parton distribution amplitude}

The two-parton light-cone distribution amplitudes for the $K_{1}$ meson are given as [26]

$$
\begin{aligned}
\left\langle 0\left|\bar{q}(y) \gamma_{\mu} \gamma_{5} q(x)\right| K_{1}\left(p^{\prime}, \lambda\right)\right\rangle & =i f_{K_{1}}^{\|} m_{K_{1}} \int_{0}^{1} d u e^{i\left(u p^{\prime} y+\bar{u} p^{\prime} x\right)}\left\{p_{\mu}^{\prime} \frac{\varepsilon^{*(\lambda)} z}{p z} \Phi_{\|}(u)+\varepsilon_{\perp \mu}^{*(\lambda)} g_{\perp}^{(a)}(u)-\frac{1}{2} z_{\mu} \frac{\varepsilon^{*(\lambda)} z}{(p z)^{2}} m_{K_{1}}^{2} g_{3}(u)\right\} \\
\left\langle 0\left|\bar{q}(y) \gamma_{\mu} q(x)\right| K_{1}\left(p^{\prime}, \lambda\right)\right\rangle & =-i f_{K_{1}}^{\|} m_{K_{1}} \epsilon_{\mu \nu \rho \sigma} \varepsilon^{*(\lambda)} p^{\rho} z^{\sigma} \int_{0}^{1} d u e^{i\left(u p^{\prime} y+\bar{u} p^{\prime} x\right)} \frac{g_{\perp}^{(v)}(u)}{4} .
\end{aligned}
$$


Here $\Phi_{\|}(u)$ is the leading twist-2 distribution amplitude, which can be expanded in Gegenbauer moments as we did in the numerical analysis (cf. Sec. IV). Note that $g_{\perp}^{(a)}(u), g_{\perp}^{(v)}(u)$ are twist-3, while $g_{3}(u)$ are twist-4 contributions, which we did not discuss as these contributions go as $1 / m_{B}^{3}$. The matrix elements for the tensor currents up to twist-3 are given as

$$
\begin{aligned}
\left\langle 0\left|\bar{q}(y) \sigma_{\mu \nu} \gamma_{5} q(x)\right| K_{1}\left(p^{\prime}, \lambda\right)\right\rangle & =f_{K_{1}}^{\perp} \int_{0}^{1} d u e^{i\left(u p^{\prime} y+\bar{u} p^{\prime} x\right)}\left\{\left(\varepsilon_{\perp \mu}^{*(\lambda)} p_{\nu}-\varepsilon_{\perp \nu}^{*(\lambda)} p_{\mu}\right) \Phi_{\perp}(u)+\frac{m_{K_{1}}^{2} \varepsilon^{*(\lambda)} z}{(p z)^{2}}\left(p_{\mu} z_{\nu}-p_{\nu} z_{\mu}\right) h_{\|}^{(t)}(u)\right\}, \\
\left\langle 0\left|\bar{q}(y) \gamma_{5} q(x)\right| K_{1}\left(p^{\prime}, \lambda\right)\right\rangle & =f_{K_{1}}^{\perp} m_{K_{1}}^{2}\left(\varepsilon^{*(\lambda)} z\right) \int_{0}^{1} d u e^{i\left(u p^{\prime} y+\bar{u} p^{\prime} x\right)} \frac{h_{\|}^{(p)}(u)}{2} .
\end{aligned}
$$

\section{APPENDIX B: HELICITY AMPLITUDES}

The polarization vectors are represented by $\varepsilon(i)$, where $i=0, \pm$ denote the longitudinal and transverse polarization of the lepton pair. The metric tensor $g_{\mu \nu}$ can be written in terms of the dilepton momenta and polarization vectors as $g_{\mu \nu}=-\sum_{i} \varepsilon_{\mu}(i) \varepsilon_{\nu}^{*}(i)+\frac{q_{\mu} q_{\nu}}{q^{2}}$. Substituting this in Eq. (55), we can write the decay amplitude as

$$
\mathcal{M}\left(B \rightarrow K_{1} \ell^{+} \ell^{-}\right)=\mathcal{L}_{\mu}(L) \mathcal{H}_{\nu}(L) g^{\mu \nu}+\mathcal{L}_{\mu}(R) \mathcal{H}_{\nu}(R) g^{\mu \nu}=-\sum_{i} \mathcal{L}(L, i) \mathcal{H}(L, i)-\sum_{i} \mathcal{L}(R, i) \mathcal{H}(R, i) .
$$

The leptonic amplitudes are easy to define. Let $\theta_{1}$ be the angle between $\ell^{-}$in the lepton pair rest frame and the $B$ meson. The angle between the $K_{1}$ meson and the lepton pair plane is $\phi$. The various leptonic amplitudes will then be given as

$$
\begin{array}{cc}
\mathcal{L}(L, 0)=2 \sqrt{q^{2}} \sin \theta_{1}, & \mathcal{L}(R, 0)=-2 \sqrt{q^{2}} \sin \theta_{1}, \\
\mathcal{L}(L,+)=-2 \sqrt{2} \sqrt{q^{2}} \sin ^{2} \frac{\theta_{1}}{2} e^{i \phi}, & \mathcal{L}(R,+)=-2 \sqrt{2} \sqrt{q^{2}} \cos ^{2} \frac{\theta_{1}}{2} e^{i \phi}, \\
\mathcal{L}(L,-)=-2 \sqrt{2} \sqrt{q^{2}} \cos ^{2} \frac{\theta_{1}}{2} e^{-i \phi}, & \mathcal{L}(R,-)=-2 \sqrt{2} \sqrt{q^{2}} \sin ^{2} \frac{\theta_{1}}{2} e^{-i \phi} .
\end{array}
$$

The hadronic amplitudes for the three polarization states are given as

$$
\begin{aligned}
\mathcal{H}(L, 0)= & \frac{G_{F} V_{t b} V_{t s}^{*} \alpha}{8 \sqrt{2} \pi m_{K_{1}} \sqrt{q^{2}}}\left(2 C_{7}^{\mathrm{eff}} m_{b}\left[\frac{\lambda T_{3}\left(q^{2}\right)}{m_{B}^{2}-m_{K_{1}}^{2}}-\left(3 m_{K_{1}}^{2}+m_{B}^{2}-q^{2}\right) T_{2}\left(q^{2}\right)\right]\right. \\
& \left.+\left(C_{9}^{\mathrm{eff}}-C_{10}\right)\left[\left(m_{B}-m_{K_{1}}\right)\left(m_{K_{1}}^{2}-m_{B}^{2}+q^{2}\right) V_{1}\left(q^{2}\right)+\frac{\lambda V_{2}\left(q^{2}\right)}{m_{B}-m_{K_{1}}}\right]\right), \\
\mathcal{H}(R, 0)= & \frac{G_{F} V_{t b} V_{t s}^{*} \alpha}{8 \sqrt{2} \pi m_{K_{1}} \sqrt{q^{2}}}\left(2 C_{7}^{\mathrm{eff}} m_{b}\left[\frac{\lambda T_{3}\left(q^{2}\right)}{m_{B}^{2}-m_{K_{1}}^{2}}-\left(3 m_{K_{1}}^{2}+m_{B}^{2}-q^{2}\right) T_{2}\left(q^{2}\right)\right]\right. \\
+ & \left(C_{9}^{\mathrm{eff}}+C_{10}\right)\left[\left(m_{B}-m_{K_{1}}\right)\left(m_{K_{1}}^{2}-m_{B}^{2}+q^{2}\right) V_{1}\left(q^{2}\right)+\frac{\lambda V_{2}\left(q^{2}\right)}{\left.\left.m_{B}-m_{K_{1}}\right]\right),}\right. \\
\mathcal{H}(L,+)= & \frac{G_{F} V_{t b} V_{t s}^{*} \alpha}{4 \sqrt{2} \pi m_{K_{1}} \sqrt{q^{2}}}\left(2\left[C_{7}^{\mathrm{eff}} m_{b} \sqrt{\lambda} T_{1}\left(q^{2}\right)-C_{7}^{\mathrm{eff}} m_{b}\left(m_{B}^{2}-m_{K_{1}}^{2}\right) T_{2}\left(q^{2}\right)\right]\right. \\
& \left.+\left(C_{9}^{\mathrm{eff}}-C_{10}\right) q^{2}\left[\frac{\sqrt{\lambda} A_{2}\left(q^{2}\right)}{m_{B}-m_{K_{1}}}-\left(m_{B}-m_{K_{1}}\right) V_{1}\left(q^{2}\right)\right]\right), \\
\mathcal{H}(L,-)= & \frac{G_{F} V_{t b} V_{t s}^{*} \alpha}{4 \sqrt{2} \pi m_{K_{1}} \sqrt{q^{2}}}\left(2\left[-C_{7}^{\mathrm{eff}} m_{b} \sqrt{\lambda} T_{1}\left(q^{2}\right)-C_{7}^{\mathrm{eff}} m_{b}\left(m_{B}^{2}-m_{K_{1}}^{2}\right) T_{2}\left(q^{2}\right)\right]\right. \\
& \left.+\left(C_{9}^{\mathrm{eff}}-C_{10}\right) q^{2}\left[-\frac{\sqrt{\lambda} A_{2}\left(q^{2}\right)}{m_{B}-m_{K_{1}}}-\left(m_{B}-m_{K_{1}}\right) V_{1}\left(q^{2}\right)\right]\right)
\end{aligned}
$$




$$
\begin{aligned}
\mathcal{H}(R,+)= & \frac{G_{F} V_{t b} V_{t s}^{*} \alpha}{4 \sqrt{2} \pi m_{K_{1}} \sqrt{q^{2}}}\left(2\left[C_{7}^{\mathrm{eff}} m_{b} \sqrt{\lambda} T_{1}\left(q^{2}\right)-C_{7}^{\mathrm{eff}} m_{b}\left(m_{B}^{2}-m_{K_{1}}^{2}\right) T_{2}\left(q^{2}\right)\right]\right. \\
& \left.+\left(C_{9}^{\mathrm{eff}}+C_{10}\right) q^{2}\left[\frac{\sqrt{\lambda} A_{2}\left(q^{2}\right)}{m_{B}-m_{K_{1}}}-\left(m_{B}-m_{K_{1}}\right) V_{1}\left(q^{2}\right)\right]\right) \\
\mathcal{H}(R,-)= & \frac{G_{F} V_{t b} V_{t s}^{*} \alpha}{4 \sqrt{2} \pi m_{K_{1}} \sqrt{q^{2}}}\left(-2\left[C_{7}^{\mathrm{eff}} m_{b} \sqrt{\lambda} T_{1}\left(q^{2}\right)-C_{7}^{\mathrm{eff}} m_{b}\left(m_{B}^{2}-m_{K_{1}}^{2}\right) T_{2}\left(q^{2}\right)\right]\right. \\
& \left.+\left(C_{9}^{\mathrm{eff}}+C_{10}\right) q^{2}\left[-\frac{\sqrt{\lambda} A_{2}\left(q^{2}\right)}{m_{B}-m_{K_{1}}}-\left(m_{B}-m_{K_{1}}\right) V_{1}\left(q^{2}\right)\right]\right)
\end{aligned}
$$

\section{APPENDIX C: HARD-SPECTATOR CORRECTION TO $V_{2}\left(q^{2}\right)$}

For heavy-to-light meson matrix elements in Eq. (29), substituting $\mathcal{M}^{K_{1}}$ from Eq. (30) and the $\phi_{+}^{B}\left(l_{+}\right)$term of $\mathcal{M}^{B}$ from Eq. (31) along with the hard scattering amplitude

$$
\mathcal{T}_{i j k l}^{\mu}=-\left[\gamma^{\mu} \frac{\not h_{-}}{4 \bar{u} l_{+} m_{b} E_{F}} \gamma_{\eta}\right]_{i j}\left[\gamma^{\eta}\right]_{k l},
$$

we arrive at the following relation:

$$
\left\langle K_{1}\left(p^{\prime}, \varepsilon^{*}\right)\left|\bar{q} \gamma^{\mu} b\right| \bar{B}(p)\right\rangle_{H S A}=-\frac{4 \pi \alpha C_{F}}{N_{c}}\left(\frac{-i}{4}\right)\left(\frac{-i f_{B} m_{B}}{8}\right)\left(\frac{1}{4 m_{b} E_{F}}\right) \int_{0}^{1} d u \int_{0}^{\infty} d l_{+} \frac{1}{\bar{u} l_{+}} * \operatorname{Tr}[\cdots]
$$

where the trace is

$$
\operatorname{Tr}[\cdots]=\operatorname{Tr}\left[\left(f_{K_{1}}^{\perp} \phi_{\perp}^{K_{1}}(u) \notin^{*} \not \not^{\prime}+f_{K_{1}}^{\|} \phi_{\|}^{K_{1}}(u) \frac{m_{K_{1}}}{E_{F}}\left(\varepsilon^{*} \cdot v \not p^{\prime}\right)\right)\left(-\gamma^{\mu} \not_{-} \gamma_{\eta}\right)\left(\phi_{+}^{B}\left(l_{+}\right)(1+\not b) \not h_{+} \gamma_{5} \gamma^{\eta}\right)\right]
$$

We solve the trace to get

$$
\operatorname{Tr}[\cdots]=-\frac{8 m_{K_{1}}^{2}}{E_{F}} f_{K_{1}}^{\perp} \phi_{\perp}^{K_{1}}(u) \phi_{+}^{B}\left(l_{+}\right)\left(\varepsilon^{*} \cdot v\right) n_{-}^{\mu}-32 f_{K_{1}}^{\|} \phi_{\perp}(u) \phi_{+}^{B}\left(l_{+}\right)\left(\varepsilon^{*} \cdot v\right) \frac{m_{K_{1}} \Delta}{E_{F}} n_{-}^{\mu} .
$$

The leading twist moments $\phi_{\perp, \|}^{K_{1}}(u)$ and $\phi_{+}^{B}\left(l_{+}\right)$are integrated and given as in Eqs. (42) and (43), respectively. Substituting Eq. (C4) in Eq. (C2) and comparing it to the first line in Eq. (2) will give us the desired result of Eq. (37). The first contribution goes like $m_{K_{1}}^{2}$, which will be multiplied by $q^{2}$ upon comparison with Eq. (2) and hence can be omitted. A similar technique can be followed for the calculation of the hard-spectator corrections to the rest of the form factors.

[1] J. Albrecht, F. Bernlochner, M. Kenzie, S. Reichert, D. Straub, and A. Tully, arXiv:1709.10308.

[2] J. Albrecht, S. Reichert, and D. van Dyk, Int. J. Mod. Phys. A 33, 1830016 (2018).

[3] N. Gubernari, A. Kokulu, and D. van Dyk, J. High Energy Phys. 01 (2019) 150.

[4] N. Isgur and M. B. Wise, Phys. Lett. B 232, 113 (1989).

[5] N. Isgur and M. B. Wise, Phys. Lett. B 237, 527 (1990).

[6] M. Neubert, Phys. Rep. 245, 259 (1994).
[7] J. Charles, A. Le Yaouanc, L. Oliver, O. Pene, and J. C. Raynal, Phys. Rev. D 60, 014001 (1999).

[8] A. G. Grozin and M. Neubert, Phys. Rev. D 55, 272 (1997).

[9] H. Georgi, Phys. Lett. B 240, 447 (1990).

[10] M. Beneke and T. Feldmann, Nucl. Phys. B592, 3 (2001).

[11] M. Beneke, T. Feldmann, and D. Seidel, Nucl. Phys. B612, 25 (2001).

[12] H. Hatanaka and K. C. Yang, Phys. Rev. D 77, 094023 (2008); 78, 059902(E) (2008). 
[13] M. A. Paracha, I. Ahmed, and M. J. Aslam, Eur. Phys. J. C 52, 967 (2007).

[14] Y. Li, J. Hua, and K. C. Yang, Eur. Phys. J. C 71, 1775 (2011).

[15] W. L. Ju, G. L. Wang, H. F. Fu, Z. H. Wang, and Y. Li, J. High Energy Phys. 09 (2015) 171.

[16] F. Falahati and A. Zahedidareshouri, Phys. Rev. D 90, 075002 (2014).

[17] S. Momeni and R. Khosravi, Eur. Phys. J. C 78, 805 (2018).

[18] S. Momeni and R. Khosravi, Phys. Rev. D 96, 016018 (2017).

[19] Z.-R Huang, M. Ali Paracha, I. Ahmed, and C.-D. Lu, arXiv: 1812.03491.

[20] K. C. Yang, Phys. Rev. D 78, 034018 (2008).

[21] H. Hatanaka and K. C. Yang, Phys. Rev. D 78, 074007 (2008).
[22] D. Ebert, R. N. Faustov, and V. O. Galkin, Phys. Rev. D 64, 094022 (2001).

[23] C. W. Bauer, D. Pirjol, and I. W. Stewart, Phys. Rev. D 67, 071502 (2003).

[24] Arslan Sikandar, M. Jamil Aslam, I. Ahmed, and S. Shafaq (to be published).

[25] C. W. Bauer, S. Fleming, D. Pirjol, and I. W. Stewart, Phys. Rev. D 63, 114020 (2001).

[26] K. C. Yang, Nucl. Phys. B776, 187 (2007).

[27] B. Aubert et al. (BABAR Collaboration), Phys. Rev. D 80, 111105 (2009).

[28] M. Beneke and J. Rohrwild, Eur. Phys. J. C 71, 1818 (2011).

[29] A. J. Buras and M. Munz, Phys. Rev. D 52, 186 (1995).

[30] R. H. Li, C. D. Lu, and W. Wang, Phys. Rev. D 79, 094024 (2009). 